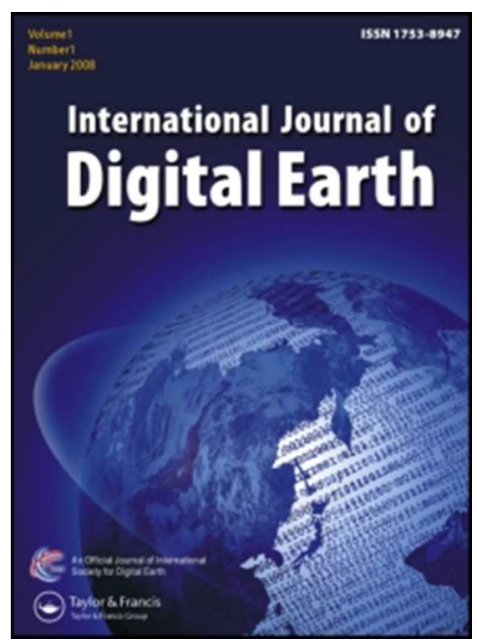

\title{
Shaping Digital Earth Applications through Open Innovation - Setting the Scene for a Digital Earth Living Lab
}

\begin{tabular}{|r|l|}
\hline Journal: & International Journal of Digital Earth \\
\hline Manuscript ID: & Draft \\
\hline Manuscript Type: & Special Issue Paper \\
\hline Keywords: & $\begin{array}{l}\text { Sustainable development, open innovation, stakeholder engagement, living } \\
\text { laboratories, virtual globes }\end{array}$ \\
\hline \multicolumn{2}{|l}{} \\
\hline
\end{tabular}

\section{SCHOLARONE}

Manuscripts 


\title{
Shaping Digital Earth Applications through Open Innovation - Setting the Scene for a Digital Earth Living Lab
}

\begin{abstract}
Science and policy increasingly request for sustainable development and growth. Similarly, Digital Earth undergoes a paradigm shift to an open platform that actively supports user engagement. While the public becomes able to contribute new content, we recognize a gap in user-driven validation, feedback and requirements capture, and innovative application development. Rather than defining Digital Earth applications top down - we see a need for methods and tools that will help building applications bottom up and driven by community needs. These should include a technology toolbox of geospatial and environmental enablers, which allow to access functional building blocks and content in multiple ways, but - equally important - enable the collaboration within partially unknown stakeholder networks. The validation and testing in real-life scenarios will be a central requirement when approaching the Digital Earth 2020 goals, which were articulated recently. We particularly argue to follow a Living Lab approach for co-creation and awareness rising in relation to environmental and geospatial matters. We explain why and how such a Digital Earth LIving lab (DELI) could lead to a sustainable approach for developing, deploying and using Digital Earth applications, and suggest a paradigm shift for Virtual Globes becoming forums for research and innovation.
\end{abstract}

Keywords: sustainable development, open innovation, stakeholder engagement, living laboratories, governance methodologies, virtual globes

\section{Introduction}

As response to the economic crisis, science and policy around the world began to reenforce the importance of user and market needs, and requested tighter integration of Research and Development (R\&D) with intended - but also more general - stakeholder networks. In Europe, for example, this approach manifested itself in the concepts of smart sustainable and inclusive growth, as part of the Europe 2020 Strategy (EC, 2010a). Location has been widely recognized as an integral factor for developing long requested multi-disciplinary applications for meeting the strategic goals (Craglia et al., 
2012a), and Digital Earth (DE) has been set into context (Craglia et al., 2012b).

Before going into any details, we should be aware of the brief overall scenery, as sketched in Figure 1. So far, policy is addressing the required needs from one angle; research from another; and users - here talking about the set of all potential stakeholders, especially the public and private citizen - are relatively disconnected from both. This picture might look equally discouraging as provocative at the first glance, but in fact it helps us to identify the gap between all three components (policy, research and users) as a required working area. We strongly believe that exactly this current gap provides us with an urgently required space for innovation, which could create economic growth as well as social benefit.

[Figure 1. Introduction to the innovation space - about here]

But, what might be the role of DE, and what is a DE application in the first place? According to Al Gore's initial vision, DE would be a knowledge store related to scientific and cultural information. According (DE) applications would focus on knowledge discovery and access, mainly by navigating a Virtual Globe. However, recent paradigm shifts in respect to this vision (Annoni et al., 2011; Craglia et al., 2012b; Goodchild et al., 2012) recognize that there is more than one Virtual Globe, and users are getting more actively engaged by providing own data and observations. In this wider scenery context, we - for the moment - consider DE applications as a pool of geospatial-inside software solutions. This notion will be revised later.

While the public becomes able to contribute with new content - e.g. Volunteered Geographic Information (VGI) (Goodchild, 2007) - we still miss some of the ingredients for innovation, particularly in terms of user-contributed validation, reflections of desirable solutions and market needs, as well as application development. While VGI is certainly the low hanging fruit - though it comes with own challenges and 
pitfalls - the real missing mechanism in getting DE applications accepted, used and providing benefit is co-creation. In order to account for community dynamics and end user needs - rather than defining DE applications top down - we see an urgent need to put the methods and tools in place that will help to build applications bottom up, driven by community demands.

As DE applications are per se evolving from an ecosystem of infrastructures and platforms, there cannot be a single governance method to the challenge mentioned above. However, we might take a structured approach on investigating the overall research and innovation ecosystem, analyzing gaps when approaching the newly set DE vision for 2020 and related political and scientific goals, and suggesting an overall governance methodology. The article at hand presents exactly this. We particularly suggest a Living Lab approach for unleashing potentials of available resources for socio-economic benefit.

The remainder of this article is structured as follows. The basic political and scientific background to this work is given in the next section, together with the currently evolving notions of open innovation and crowd sourcing. The mentioned analysis of today's innovation ecosystems and arising possibilities relating to the application of Living Laboratories (Living Labs in short) - is presented thereafter. This includes an overall framework for characterizing innovation facilities, as well as a generic governance methodology. On this basis, we apply the concept of open innovation and the Living Lab approach to the sustainable development of DE applications. Our investigations begin with a state of play analysis and then re-visit the notion of DE applications and their evolvement. Finally we suggest a Digital Earth LIving lab (DELI) as a way towards the DE 2020 vision, and the political and scientific goals relating to social benefit and economic growth. In the subsequent discussion, we 
present a SWOT (Strength, Weaknesses, Opportunities and Threads) analysis of the proposed approach and elaborate on possible future scenarios, just before drawing conclusions and defining new work items.

\section{Background}

The major incentive behind this work is sustainable development (WCDE, 1987). Recent international events - such as the 2009 Royal Society meeting on 'The sustainable planet: opportunities and challenges for science, technology and society' (http://royalsociety.org/sustainable-planet) and the 2012 Rio+20 Conference on sustainable development (http://www.uncsd2012.org) - reviewed the role of new advances in science and technology to address the grand challenges of society in the context of climate change, supply of essential materials, food and energy, and new disease patterns (Howard and Chamberlain, 2011; UNEP, 2012). As an example of successful stories, the US Environmental Protection Agency (EPA) has recently realigned its research and development activities in terms of efficiency and better decision-making processes (Anastas, 2012). EPA's new principles are sustainability, integrated inter-disciplinary research, and innovation.

In this context, the emerging field of sustainability science (Kates et al., 2001; Swart et al., 2004; Wiek et al., 2012) is paving the way to reconciling science, environmental policies, and society needs to support sustainable development. Sustainability science considers the interplay of social, economic and environmental ecosystems, amongst other reasons in order to find novel ways for balancing benefits. It should be addressed by emphasizing a close collaboration and interrelation between relevant participants such as industry, academic institutions, and citizen from a holistic and integrative perspective (Reid et al., 2010). 


\subsection{Political Context}

In line with the above, the Europe 2020 strategy (EC, 2010a), formulated by the European Commission (EC), also emphasizes a smart and sustainable growth by promoting a more resource efficient, greener and more competitive economy. The EC identifies the Digital Agenda (EC, 2010b) and Innovation Union (EC, 2010c)) as flagship initiatives and main drivers to address these challenges, where technologicalintensive domains are crucial to develop innovative and sustainable applications. These examples underline the same central message: innovations in Information and Communication Technologies (ICT) and scientific advancements are common denominators to enable sustainability development, regardless of particular scientific disciplines.

This is complemented by social challenges or benefit areas, which are on European level reflected in the Horizon 2020 (EC, 2011) - including the challenges of health, demographic change and wellbeing, smart, green and integrated transport, and climate action, resource efficiency and raw materials -, as well as globally - at least for the geospatial community - by the Group on Earth Observation (GEO, http://www.earthobservations.org). GEO’s Social Benefit Areas (SBAs), for example include the improving the management of energy resources, and the improving weather information, forecasting and warning.

\subsection{Science Context}

Within the scope of their 'Grand Challenges in Global Sustainability Research' analysis, also the International Council for Science (ICSU) has emphasized that the understanding and management of climate change, environmental degradation, and the quest for sustainable growth are among the most fundamental challenges 
facing humanity in the 21 st century (ICSU, 2010). As seen earlier, an important driver towards sustainability science is the establishment of a multi-disciplinary, integrated and collaborative approach. Amongst others, Hey and Trefethen (2005) propose the use of research (cyber-)infrastructure ${ }^{1}$ to support the needs of multidisciplinary collaborative research by sharing distributed resources - e.g. datasets, algorithms - among science teams. Geospatial cyber-infrastructures (Yang et al., 2010; Wright and Wang, 2011), regarded as cyber-infrastructures that focus particularly on geospatial resources, support processing capabilities for end users such as geospatial analysis, environmental assessment and modeling, decision making, and policy impact. The integration and use of accurate, up-to-date geospatial data through geospatial cyberinfrastructures leverages efficient management, sharing and exploitation of geospatial resources scattered among numerous agencies and institutions. The next generation of cyber-infrastructures should be able to manage the complexity of natural processes and environmental changes, so that scientists may understand and predict them accordingly (Shupeng and van Genderen, 2008). Some promising examples are EarthCube (http://earthcube.ning.com) of the National Science Foundation (NSF), GEO's Global Earth Observation System of Systems (GEOSS, http://www.earthobservations.org), and European Strategy Forum for Research Infrastructures (ESFRI, http:// ec.europa.eu/research/esfri).

With our work, we urge for a 'change to sustainability' in the development of DE applications which be able to adapt to new technologies, contribute to reduce costs,

1 The authors are well aware that the concepts research infrastructure and cyberinfrastructure might be distinguished from each other, particularly in respect to aspects of virtualization. As these distinctions are not paramount to the level of detail of the presented work, we use the 'term research' infrastructure in the following section, referring to both. 
optimize their use, and create reliable cyber-infrastructures, community networks, and real-life experimentation facilities. We see user involvement as a major driver for successfully shaping the next generation of DE applications.

\subsection{Open Innovation and Crowd Sourcing}

The scenery outlines above has been complemented by a shift of paradigm in stakeholder engagement. Open innovation and crowd sourcing are the most important concepts to mention.

A particular organization develops an open innovation strategy when it is willing to interact with stakeholders outside the closed boundaries of the organization so as to use resources - e.g. methods, ideas, knowledge, technologies - and exploit internal and external paths to market them (Chesbrough, 2003). Whereas R\&D is classically addressed 'in-house', open innovation attempts to make underlying challenges, intermediate results, and even early stages of product or service development, visible to wider audiences; in some cases even to the public.

Crowd sourcing in general describes acts of outsourcing any kind of task to some community (referred to as 'the crowd"). This outsourcing takes place in open calls or competitions that call for solutions to a more or less precisely described problem (Chesbrough, 2003). Crowd sourcing can be thus seen as one means to distribute problem solving following an open innovation program without restrictions on the targeted audience. The notion recently gained attention in the context of Web 2.0 (Howe, 2006), where collaborative technologies and techniques has greatly fostered the participation of users and citizens as an active producer of information products and new services.

Main reasons for applying crowd sourcing include: (i) the low investments to start a project, because web-based solutions to collect and capture ideas, and support 
distributed users are already in place in form of Web 2.0 web sites and tools (Howe, 2006); and (ii) the intention to capture a vast quantity of ideas, either in some topics of interests or over a broad spectrum of themes.

In general, open innovation strategies put emphasis in the collaboration, participation, and direct communication and exchanges of views with external user and stakeholder during the entire life-cycle development of a product or service. This fundamental aspect can be accomplished by crowd sourcing. It can be thus seen as one means to distribute problem solving and challenges over an unlimited targeted audience, which can participate and submit ideas and solutions. For instance, the before mentioned EPA recently launched challenges through an online web site addressed to an open audience for getting solutions to particular environmental problems (Anastas, 2012).

\section{Analysis of Innovation Ecosystems and the Living Lab Approach}

As a whole, we intent to reach the goals of sustainable development and growth. Keeping the above in mind, how may we - even independent of the DE context approach or characterize innovation ecosystems and related governance? Which approaches might be valuable for closing the gap, which we called the 'innovation space' of Figure 1?

\subsection{A Framework for Characterizing Innovation Ecosystems}

It is clear that open innovation strategies should involve a wide range of potential stakeholders in the design, development, and testing of a product or service. Ballon et al. (2005) proposed a conceptual framework for characterizing different types of innovation strategies. First, the takeoff of a product or service onto the market is mostly bound to the degree of maturity of the technology used. Second, the aim of the open 
innovation strategies may range from design and development to testing platforms. For instance, prototyping strategies are mostly focused on design and implementation aspects, whereas test beds are centered on testing and assessing products and services. Finally, the degree of openness refers to the level of 'external participation' as a product, as well as a service, goes through its life-cycle from its inception to the market. Extreme examples are closed teams such as in-house R\&D group to open innovation participation such as crowd-sourcing.

Figure 2 presents a revised version of this conceptual framework, where technology maturity and the degree of openness are on the horizontal axis, bottom and top respectively. We modified the initial graphic from Ballon and others for illustrating what we believe is currently missing - or not matured enough - in order to seriously address the innovation space between science, policy and all potential stakeholders. Accordingly, the figure underlines the current gap in technology transfer, it requests catalysts between $\mathrm{R} \& \mathrm{D}$ results and ready-to-use products in the marketplace.

[Figure 2. Existing gap to leverage R\&D results to market-ready products (typology of open innovation strategies modified from Balloon et al. (2005)) - about here]

A crowd-sourcing approach may be a low investment to support some of the open innovation strategies in Figure 2 - for instance to set up a forum to collect and capture ideas for feeding early stages of design and developing, such as prototyping and running field trials. However, due to its radical openness, crowd sourcing is not suitable for every scenario. We especially seek confident stakeholder relations and want to initiate co-creation processes following a flexible but still channeling governance model. Since Living Labs support such more controlled approach, we will focus on the concept of Living Lab as a central strategy further down. 


\subsection{Generic Governance Methodology}

As the degree of openness move towards the right side of Figure 2, we need a governance methodology to establish well-defined methods to coordinate and interrelate involved participants. Figure 3 depicts such a generic governance methodology based on the Cyclic Innovation Model (CIM) (Berkhout, 2000; Berkhout and van der Duin, 2007) and on the results of the European-funded Apollon project (http://www.apollonpilot.eu). The extended CIM is reflected in the product development part of Figure 3 (bottom). The combination of design-develop-testing phases is accompanied by a scanning of the environment (not represented in Figure 3) that is meant for enabling product launch to the market once the appropriate level of maturity is reached. As the authors highlight, the most important feature of CIM is that the different phases towards innovation are not a one-directional but cyclic: innovations build on innovations (feedback), ideas create new concepts, successes create new challenges, and failures create new insights.

[Figure 3. Overall methodology to enable customer-driven product development (adapted from Apollon project) - about here]

The Apollon project particularly suggests extending the CIM methodology to support a governance model for the creation of user-driven innovation networks. Accordingly, the methodology complements product (and service) development (bottom Figure 3) with the stakeholders consolidation (top Figure 3). The latter consists of four phases:

(1) Connect: This phase concerns the setup of a network of participant stakeholders, as well as the initial definition of a collaboration infrastructure in terms of required resources, scope, and impact of the project; trying to answer the 
following questions: Do we prefer to engage a lot of people, even without knowledge or expertise in a particular theme, or a smaller, more selective team? Do we find incremental or disruptive ideas? Do we want to establish a trusted network of partners or just to attract people interested in such themes?

(2) Plan and Engage: It defines the collaboration agreement in terms of managing Intellectual Property Rights (IPR), roles, formal agreements among the involved stakeholders, as well as the initial collection of ideas, requirements and definition of use cases. Some questions in this phase are: Who plays the role of coordinator? Who acts as facilitator? How information and conversions are channeled through the network of stakeholders?

(3) Support and Govern: It refers to the supporting tools required to begin and support the product (or service) development cycle (bottom part of Figure 3) from the initial requirements and use cases defined in the planning and engagement phase. As each open innovation program may potentially be targeted to any type of product and involve different kinds of stakeholders, the supporting tools, services, and infrastructure to govern and carry out the product development phases may be distinct in each case. Definition of milestones and assurance of active communication among stakeholders are common tasks of this phase.

(4) Manage and Track: It consists of assessing the expected impact and expectations of the outcomes of the development cycle, according to the initial goals and scope specified in the planning and engagement phase and updated through successive iterations in the product development cycle. This also includes possibly required training and the dissemination of results of the completed iteration. 
The aim of the generic governance methodology is to put stakeholders and technical staff working together from the early stages of a product or service. In principle, this methodology can be deployed at all stages of the innovation ecosystem framework introduced above (Figure 2), i.e. to realize prototypes, test beds, but also market and social pilots. In respect to the goal of our work, we will particularly focus on the use of this methodology for approaching the required catalyst between less mature and closed developments and open markets (see also Section 3.2).

\subsection{The Concept of Living Labs}

Ballon et al. (2005) already suggest a possible mechanism for implementing the required facilitator for open innovation and co-creation: Living Labs. Over the last decade this concept has been promoted as one promising way to address the above challenges, i.e. transforming available inputs (prototype software components and various technology or design focused testing facilities) with the desired outcome (mature and innovative products with strong market take up). They incorporate the requested catalyst and also - at least in parts - social and market pilots.

The term Living Lab as such is difficult to define (Ballon et al). The main defining characteristics may be summarized as follows:

- A Living Lab enables conducting experiments in familiar and real-world contexts. Experimental settings resemble as closely as possible real-life situations and embrace the uncontrollable dynamics of everyday life.

- A Living Lab provides an experimentation environment for co-creation in close collaboration with (end) users. This means that users openly and actively participate in all stages of a product development (e.g. ideas, design, development, testing), and not only in last phases. 
- Opposed to (fully-open) crowd-sourcing, a Living Lab follows an open innovation strategy where there are restrictions on the targeted participating audience.

- A Living lab can be seen as an open innovation ecosystem. By promoting cocreation, community dynamics and real life contexts, innovation processes may emerge to create new opportunities and capture unique ideas from a set of stakeholders who have valuable knowledge for shaping specific problems, and social and technological needs.

The European Network of Living Labs (ENoLL, http://www.openlivinglabs.eu) provides many more details about the overall concept and hosts searchable contact information for more than 300 Living Labs within Europe, China and Africa. Details about possible more specific governance models for Living Labs are illustratively described in (MEDLAB, 2011).

While all the above findings can be equally applied to the realm of DE as to any other, we will now, i.e. in the next section, further elaborate on the specific of DE applications and eventually required adaptations when applying the Living Lab approach in this context. In relation to existing investigations on governance for DE - as for example developed in the context of EarthCube ${ }^{2}$ - we seek a common governance methodology that allows us (i) to trigger innovation for DE applications; and (ii) to inter-connect Living Labs using the DE capabilities.

2 The EarthCube's Governance group (http://earthcube.ning.com/group/governance) is in charge of establishing a series of governance functions to support collaborative Earth science research and product development. These functions refer to desirable supporting tools, infrastructure capabilities and social agreements for enabling collaboration across multiple communities. 


\section{Open Innovation for Digital Earth Applications}

In this section, we customize the notion of Living Labs to the DE context, including a potential methodology for realizing a DE Living Lab in order to address sustainable development and growth, i.e. to boost socio-economic benefit, in relation to $\mathrm{DE}$ applications. We promote this approach for implementing the 'catalyst' requested above, particularly for closing the gap in user-contributed validation, reflections of desirable solutions and market needs, as well as development of innovative DE applications.

\subsection{Current Situation - Available Inputs}

Over the years, a large set of existing components for implementing DE applications emerged from test beds - such as OGC Web Services (OWS) or GEO/GEOSS Application Integration Pilots (AIPs) ${ }^{3}-$, or from prototypes, which have been developed by research projects - e.g. under the funding of NSF or the EC. As a prominent example, the NSF EarthCube programme aims to develop a user-communityguided cyber-infrastructure to integrate geospatial data across specialties on earth systems and foster collaboration between these (science) communities. Under the EC's FI-PPP (Future Internet Public-Private Partnership, http://www.fi-ppp.eu) Programme, several on-going research projects are pursuing to build a series of enablers for multiple usage areas - including environment (Havlik et al., 2011) - with a strong commitment on stakeholder engagement and innovation. As another example, the EU-funded EuroGEOSS project has contributed to the GEOSS Common Infrastructure (GCI) with concrete and valuable operational components in support of technology test beds,

\footnotetext{
${ }^{3}$ It should be noted that we face a terminological issue here, related to Figure 2, an AIP is a test bed, because the produced results are not market ready.
} 
GEOSS AIP or even development of cross-thematic environmental applications

(Vaccari et al., 2012). In the context of the INSPIRE Directive (EC, 2007), harmonised data models (INSPIRE Data Specifications) have been tested by the community, and prototypes for assessing INSPIRE Networking Services and supporting tools have been developed. Both can be seen as initial inputs for a Re-usable INSPIRE Reference Platform (see also http://ec.europa.eu/isa/actions/01-trusted-information-exchange/117action_en.htm). Together with many other, all those components provide data and service specifications; reference models; software tools together with patterns and guidelines of using them; and existing test facilities, platforms and technical infrastructures.

These building blocks are complemented by an increasing number of Virtual Globes, such as Google Earth (http://earth.google.com), NASA's World Wind (http://worldwind.arc.nasa.gov), Microsoft's Bing Maps (http://www.bing.com/maps) and Esri's ArcGIS Explorer (http://www.esri.com/software/arcgis/explorer). All in all can be summarized as 'toolboxes' or enablers, which ensure a technology push towards DE applications.

\subsection{Digital Earth Applications Re-Visited-Desired Outputs/Results}

Before we can seriously address the governance question for the sustainable development of DE applications, we should examine what finally constitutes a DE application. In other words, we should examine the application-pull which opposes the above mentioned push of technology.

Instead of looking into the available applications of today, or expanding all possible ways of applying the available tool box(es), we suggest addressing this issue bottom up by examining requests for DE products, which then derive requirements towards the toolbox of DE components, or (environmental and geospatial) enablers, as 
we called them above. Once this has been clarified, we start investigating approaches for realizing this, and at the same time improving available components for implementation (see Section 4.3).

At a generic level, we should be able to distinguish what qualifies any given application in order to be a DE application. Initially, we will require some form of spatial-temporal characteristic, i.e. involving information resources which have some form of location information related to them, or offering any form of geospatial processing functionality. Additionally, we require some capability for visualization on a Virtual Globe. This second condition is introduced in order to keep focus and to distinguish any 'geospatial-inside' software from a real DE application.

It should be noted, that whereas we have only one planet earth, there are - and always will be - multiple Virtual Globes available, all allowing for diverse (user) perspectives (Craglia et al., 2012b). Still, diverging from Al Gore's initial vision of a DE - in the end - Virtual Globes will only provide the background layers and the environment of offering value added services for collaboration, in the sense of a Platform as a Service (PaaS) (Raines and Pizette, 2010). It is the dependency on the responding users, which will define the success and evolvement of a DE application.

We do have some tools available today. Nevertheless, we could dramatically boost stakeholder uptake, mainly because DE application development still requires complex development skills and is neither intuitive nor easy accessible to layman. The Eye on Earth platform (http:// watch.eyeonearth.org) provides a good example. The system follows a sophisticated concept in making environmental information not only available to users, but also to provide additional information and to extend the platform if desired. Eye on Earth is running and expanding in scope. Still, development efforts and private contributions are done by a few individuals without a large external driving 
force. The full potential might be unleashed by introducing an according governance methodology and open community.

In following the above, Virtual Globes will in the end not be the DE application in itself, but become forums for collaborative DE application development (see also (Schade et al., 2010)). These globes will have to offer lightweight and easy development tools, similar to citySDK (http://www.forumvirium.fi/en/projectareas/smart-city/citysdk) in the context of smart cities or the goals of the SATIN project (http://www.satinproject.eu/team) on easy app development for mobile end devices. Underlying innovation processes should be supported by incubators for showcasing new developments, but also with market places - or a single market place, which is shared across Virtual Globes - for operational products.

We certainly could further philosophies about what then makes DE, e.g. the collection of all DE applications, but also for brevity waive this opportunity here.

\subsection{DELI as a Way Forward}

As a direct consequence of the discussions on open innovation, crowd sourcing and Living Labs, we suggest approaching the desired outputs (Section 4.2) by taking the available inputs (Section 4.1) and applying the Living Lab approach to the development of DE applications. We call this the Digital Earth Living Lab (DELI).

DELI complements the virtual/digital world with grounding in the reality, i.e. linking back to regions on planet earth in which the social and economic benefits of DE applications can be tested and exploited. These regions might, for example, be cities or rural areas - both of varying size - and the might cross administrative borders.

In order to account for community dynamics and real end user needs, a sound and flexible methodology is required to help us to shape a sustainable and open innovation ecosystems to materialize DELI. As the methodology of choice, we decided 
to follow the governance methodology outlined above (Section 3.2). Before applying this methodology, we should investigate the adaptations that are required for DELI. We will do this below, by re-visiting every step and elaborating on the specific objectives of each.

\subsubsection{Connect in DELI}

The initial seed for a DE application is the intended scope, resources and motivation. The starting context is accompanied with a scanning of the current state-of the art, searching specially for related prototypes and test beds - e.g. the Re-usable INSPIRE Reference Platform, GEO GCI, the EarthCube cyber-infrastructure, and results from R\&D projects -, and so on which may be potentially inputs for DELI.

Initial conversations and contacts are primary to establish a network of interested stakeholders around the initial motivation and intended objective of DELI. Accordingly, a kind of advisory board might participate from the beginning, seen as key actors coming from related government organizations - including National Government Organizations (NGOs) -, big industrial players, Small and Medium Enterprises (SMEs), standardization bodies and enthusiastic citizen. Criteria for delimiting the initial set of stakeholders depend on envisioned individual DE applications and are mostly determined by the scope and the sought impact in terms of either incremental or disruptive innovation. One promising research focus could be the combination of Open Government Data (Perego et al., 2012) with VGI (De Longueville et al., 2010).

\subsubsection{Plan and Engage in DELI}

Having initiated first contacts to potential stakeholders, the next step goes further, to engage and consolidate a trusted network of stakeholders. This means to explicitly define the collaboration agreement - e.g. IPR management, formal agreements and 
liaisons - among the involved participants and the definition of project roles and basic collaboration tools, such as mailing lists and wiki sites, but also Virtual Globes. The ultimate goal is to make clear communication protocols and information channels to enable open, timely, and fluid conversations between the stakeholder network and the ICT staff and developers of DE applications, so as to form a trusted community.

One of the first commitments of the trusted community is the elaboration of a plan by collecting ideas, requirements and agreement on common and representative use cases, based on the initial scope and motivation defined in the earlier phase. This implies for instance to start from existing developments in the target themes of desired DE applications - such as GEO GCI as a reference model, observations available from Eye on Earth, INSPIRE data sets and services, etc. - intertwined with community discussions (e.g. stakeholder workshops) towards the identification of target markets and sustainable best practices for further developing phases.

\subsubsection{Support and Govern in DELI}

This step is likely the most critical part in DELI because it is aimed to smoothly fit the stakeholder network together with the DE applications development itself. That is, stakeholders actively participate to turn a traditional product development process into a co-design, co-development and co-testing process in which unique, innovative ideas may naturally arise. These co-creation processes should be thought of as incremental iterations towards marketable DE applications. Co-testing conducted in real-world situations - beginning in local and regional settings and to scale to cross-border scenarios if required - should lead to new ideas, needs and emerging technologies which become in valuable feedback for co-design and co-development processes. As the DE application gets closer to a market-ready product, potential industry should be attracted, for instance, as observers as part of the stakeholders networks. 
The realization of co-operative design, development and testing processes require supporting infrastructures (Zheng et al., 2011), which especially should include research infrastructures, such as those spearing from ESFRI, as detailed in Section 2.2, but also infrastructures for collaboration. In the particular case of DE applications, the connection to research infrastructures might be ensured via the International Society for Digital Earth (ISDE) or by a complementing body such as the suggested European or even International Digital Earth research Network (EDEN or IDEN, respectively) (Annoni et al., 2011). Virtual Globes have to be equipped with collaboration tools - as for example, access to environmental and geospatial toolboxes via standardized software development kits (SDKs), incubators for community generated DE applications or access to a shared market place. Still, the scientific aspects clearly miss competences from the behavioral science on user dynamics. Application design and serious gaming should become closely connected in the loop.

\subsubsection{Manage and Track in DELI}

The promotion to the market is subject to an assessment of the expected impacts and expectations of the DE applications. Responsible management and steering committees might be defined out of the existing ISDE, become an additional role of the suggested IDEN, or be newly set up. It might also make sense to establish a network of regional bodies in order to more particularly account for location-dependent markets and cultures.

In any case, decision-making processes must be transparent to keep the stakeholder community informed of the impact results. Indeed, such results may become the seed for new DE applications, starting again the connect phase in DELI. This will certainly benefit from and improve with each iteration of the cyclic governance methodology as introduced in Section 3. 


\subsubsection{Summary of the Extended CIM in DELI}

Figure 4 summarizes the resulting situation, in which currently available inputs research prototypes, results from field trials and technical components out of test beds (lower-left part of the figure) - serve as the seeds for co-design, co-development and cotesting following the extended CIM within DELI (lower-central part). Outputs of DELI are illustrated towards the lower-right; those should be matured DE applications close to market release. The increased opening of the product, but also the co-creation processes is illustrated on the top part of the illustration. Increasing amounts of stakeholders become involved with each iteration. Talking about Virtual Globes in this context, they could be overlaid with the central part of the figure, as the platforms/forums facilitating the co-creation processes and governance for $\mathrm{DE}$ as a whole. We omitted this additional graphic element in order to not overload the figure.

[Figure 4. Open innovation ecosystem for DELI - about here]

\section{Discussion}

Having so far only introduced the overall concepts, we now concretize the possible shaping of DELI and of future DE applications. We first provide a Strength, Weaknesses, Opportunities, and Threads (SWOT) analysis (McKeown, 2012), in order to foresee the intended role of Living Lab(s) in particular and open innovation ecosystems in general in creating next-generation DE applications. This is followed by several sketches of potential future scenarios of DELI taking shape and creating impact.

\subsection{SWOT Analysis of DELI}

A DELI focused SWOT analysis - i.e. acknowledging general characteristics of the Living Lab approach, but projecting them particularly on DE applications - reveals the 
following.

Strength:

- Direct involvement of DE stakeholders, including citizen, in the design, development and testing of DE applications.

- Quick to respond to changing expectations on and needs of DE applications.

- High probability of addressing real social or economic benefit by produced DE applications.

- Ability to capture and understand local and regional settings into DE applications.

- Capacity to scale from local and regional settings to wider levels or cross-border scenarios.

Weaknesses:

- Missing expertise in the current DE application development domain, particularly on carrying research into innovation.

- Compared to conventional DE application production, considerable more effort required for continuous user engagement and monitoring.

- High entry barriers to Virtual Globes and the associated tools, i.e. great skills required.

- Currently no harmonized governance methodology for establishing and running DELI put into practice.

Opportunities:

- Bringing DE applications (improved version of current prototypes and newly emerging ideas) to society or targeted market. 
- Expanding DE applications into new markets (e.g. smart cities) or thematic markets (e.g., health, demographics).

- Creating a DE applications market on its own (to gain visibility)

- Creating growth by sustainable DE application production.

- Promoting current (geospatial) information infrastructures and (more generally, research infrastructures a foundation for wider multi-purpose platforms for science-stakeholder collaboration.

Threads:

- Lack of training, education or even culture of open innovation.

- Losing control of DE application production.

- Proficient handling of IPR and branding of created DE products.

- Marketing of DE applications in real-life scenarios.

- Cost of developing common methodology and applying it at diverse experimentation sites.

This analysis reveals several central aspects, particularly the need to leverage training, education and enterprise mindset towards open innovation. We certainly will require a cultural shift in the attitude of many current tool providers and of numerous stakeholders. The required resources for carrying this still have to be identified and freed. The same applies for the required controlling (or better channeling bodies). Clear mechanisms for ensuring IPR and security have to be put into place in order to enable DELI as a whole. 


\subsection{Envisioned Future Scenarios}

A logical next step after completing the SWOT analysis would be the establishment of an action plan for implementing and maintaining DELI. However, due to the novelty of the concept and further required elaborations, we first see the need to depict possible scenarios to which DELI could lead us. How might these change DE applications and the use of Virtual Globes in the (near) future? We depict possible answers below. Each of the following six paragraphs sketches one possible scenario, with increasing level of sophistication and effort required.

Do nothing scenario. If we continue in the common way, drastically speaking, we will end up in a setting of scattered Virtual Globes, diverse and disconnected tool boxes - i.e. environmental and geospatial data and services, together with prototypes and detached operational applications - and diverse marketplaces; facing the risk of losing identity and the branding of DE applications. To this end, users will be disconnected and confused and we fail reaching sustainable development.

DELI as a single laboratory. History should tell us that, trying to realize the proposed approach in a monolithic system, i.e. on one Virtual Globe - say Bing Maps and trying to connect available inputs in a single prescribed manner - i.e. offering off the shelf deployment mechanisms of toolboxes and DE applications on Bing Maps only - will eventually fail. One accompanying big Living Lab - grounding Bing Maps in reality - would face issues of scaling local and regional trials to world-wide coverage. Furthermore, we would miss opportunities for widening perspectives for approaching the revised DE vision, due to lack of alternatives.

DELI as a network of (thematic) Living Labs. Scaling and cross-border scenarios could be reached by connecting - i.e. networking - Living Labs, which work on similar themes - such as the impacts of climate change, environmental impacts on health etc. and are located at different places across the globe. However, as we should have learned 
from the past, such approaches result in silos. In other words this solution might end up in de-coupled thematic clusters of DE applications on one of more Virtual Globes - e.g. Bing Maps for climate change, Google Earth for smart transport, and NASA world wind for eco-energy and well-being. By neglecting that thematic areas are usually dependent from each other, this complete contradicts the growing request for multi-disciplinary approaches.

DELI as a network of (transversal) Living Labs. Networking transversal Living Labs - i.e. those which do not focus on a particular theme, but primarily on enabling experimentation facilities and collaboration enablers - is certainly an approach for addressing the concerns of above, and could also lead to interconnected Virtual Globes or portable DE applications. For example, Living Labs around the globe might support the cyclic governance method for DE application development on Bing Maps, Google Earth and NASA World Wind. This might include approaches for common incubators for demonstrating and co-creating applications and entry points to a shared market place. However, a purely transversal solution is impossible to succeed due to missing focus and - more importantly - application needs. Missing local expertise and data on, e.g. climate change and the related environmental, social and economic phenomena will obviously block any serious testing of DE applications on climate action.

DELI as a network of thematic and transversal Living Labs. Combining the last two scenarios, might get close to a successful innovation ecosystem in the DE context. Applications for related themes could be tested as diverse places across the entire globe and the overall management could be harmonized leading to comparable results.

However, this would still miss easy connectors to the current situation (see also Section 4.3.1 for available inputs) and to possible developments of the future. Focusing on the Living Lab only, we might neglect required and important testing of toolboxes on a 
more technical level, activities such as OGC OWS, GEO AIPs or the testing of the INSPIRE Data Specifications, would not be supported per se, due to the Living Lab focus.

DELI as networked experimentation sites. In an ideal and equally realistic setting, instead of considering DELI as a Living Lab in isolation, it becomes well integrated into other testing and experimentation approaches and the implementing facilities. New ideas, prototypes and DE application products will undergo a life-cycle covering the complete ecosystem framework (Figure 2). For example, INSPIRE Data Specification testing can happen in test beds, prototypes on INSPIRE Networking Services can be established, and then the result of both together might be fed into a Living Lab context, which stimulates innovation based on solid scientific, political and technical grounds, and thereby facilitate at least Europe wide implementation and maintenance of INSPIRE (see also (Schade and Granell, 2012)). Only such a situation can fully implement the catalyst requested in Section 3 and thereby help to leverage the potential socio-economic benefits of the innovation space as introduced in the first section of this article.

\section{Conclusions and Future Work}

In this paper, we - for the first time - connect the concept of DE and Living Labs. We did so, because of (i) the overall need for sustainable development and growth; (ii) the identified high potential of the innovation space between science - policy and users;

(iii) and the integrative nature of geospatial information. We particularly highlighted the potential for sustainable DE application development and sketched possibilities to adapt existing building blocks, including methodologies, enabling toolboxes and research infrastructures, as enablers for establishing a Digital Earth Living Lab - DELI. DELI would ground the virtual world of DE to facilitate co-creation, validation and testing, 
and thereby fostering open innovation for future generations of DE applications. Socioeconomic benefit has been defined as the central goal.

The scenery presented in this paper will be the seed for a series of related activities. As a subsequent step, we will elaborate on the required action plan for realizing DELI. The accompanying governance framework - following a cyclic methodology - will be a driving factor.

\section{References}

Anastas, P. T., 2012. Fundamental Changes to EPA's Research Enterprise: The Path Forward. Environmental Science \& Technology 46 (2), 580-586.

Annoni, A., Craglia, M., Ehlers, M., Georgiadou, Y., Giacomelli, A. et al., 2011. A European perspective on Digital Earth. International Journal of Digital Earth 4(4), 271-284.

Ballon, P., Pierson, J., Delaere, S., 2005. Test and experimentation platforms for broadband innovation: examining European practice. In Conference Proceedings of 16th European Regional Conference by the International Telecommunications Society (ITS), Porto, Portugal, September, 2005.

Berkhout, A.J., 2000. The dynamic role of knowledge in innovation: An Integrated Framework of Cyclic Networks for the Assessment of Technological Change and Sustainable Growth. Delft University Press, Delft.

Berkhout, A.J. and van der Duin, Patrick A., 2007. New ways of innovation: an application of the cyclic innovation model to the mobile telecom industry. Int. J. Technology Management 40(4), 294-309.

Chesbrough, H.W., 2003. Open Innovation: The new imperative for creating and profiting from technology. Boston: Harvard Business School Press.

Craglia M., Nativi, S., Diaz, L. and Vaccari, L., 2012a. Towards Multi-Disciplinary 
Interoperability: the EuroGEOSS contribution. EuroGEOSS Conference, Madrid, Spain, January 2012.

Craglia, M., de Bie, K., Jackson, D., Pesaresi, M., Remetey-Fülöpp, G., et al., 2012b. Digital Earth 2020: towards the vision for the next decade. International Journal of Digital Earth 5(1), 4-21.

De Longueville, B., Annoni, A., Schade, S., Ostlaender, N. and Whitmore, C., 2010. Digital Earth's Nervous System for crisis events: real-time Sensor Web Enablement of Volunteered Geographic Information. International Journal of Digital Earth $3(3), 242-259$.

EC, 2007. Establishing an infrastructure for spatial information in the European Community (INSPIRE), Directive 2007/2/EC of the European Parliament and of the Council, 14 March 2007.

EC, 2010a. EUROPE 2020 - A strategy for smart, sustainable and inclusive growth. COM(2010) 2020, March 2010.

EC 2010b. A digital agenda for Europe. COM(2010) 245 final/2, August 2010.

EC 2010c. Europe 2020 Flagship Initiative Innovation Union. COM(2010) 546 final, October 2010.

EC 2011. Horizon 2020 - The Framework Programme for Research and Innovation. $\operatorname{COM(2011)~} 808$ final, November 2011.

Goodchild, M.F., 2007. Citizens as voluntary sensors: spatial data infrastructure in the world of Web 2.0. International Journal of Spatial Data Infrastructures Research (2) 24-32.

Goodchild, M.F., et al., 2012. Next-generation Digital Earth. Proceedings of the National Academy of Sciences 109(28), 11088-11094

Havlik, D., Schade, S., Sabeur, Z.A., Mazzetti, P., Watson, K., Berre, A.J., Mon, J.L., 
2011. From Sensor to Observation Web with Environmental Enablers in the Future Internet. Sensors 2011 (11), 3874-3907.

Hey, T., and Trefethen, A.E., 2005. Cyberinfrastructure for e-Science. Science 308, $817-821$.

Howard, J.A. K. and Chamberlain, M., 2011. The sustainable planet: opportunities and challenges for science, technology and society. Philosophical Transactions of the Royal Society A 369 (1942), 1715-1718.

Howe, J., 2006. The Rise of Crowdsourcing. Wired, June 2006.

ICSU, 2010. Grand Challenges in Global Sustainability Research: A Systems Approach to Research Priorities for the Decade. International Council for Science, Paris, France, 2010 (pre-publication version).

Kates, R. W, et al., 2001. Sustainability Science. Science 292(5517), 641-642.

McKeown, M., 2012. The Strategy Book. FT Prentice Hall.

MEDLAB, 2011. MEDLAB Governance Guidelines. Deliverable of the MEDLAB project - Component 5: Strategic Governance, Phase 1: Governance Methods, August 2011.

Perego, A., Fugazza, C., Vaccari, L., Lutz, M., Smits, P., Kanellopoulos, I, and Schade, S., 2012. Harmonization and Interoperability of EU Environmental Information and Services. IEEE Intelligent Systems, in press.

Raines, G. and Pizette, L., 2010. Platform as a Service: A 2010 Marketplace Analysis. MITRE Technicel Paper, October 2012.

Reid, W. V., Chen, D., Goldfarb, L., Hackmann, H., Lee, Y. T., Mokhele, K., Ostrom, E., et al., 2010. Earth System Science for Global Sustainability: Grand Challenges. Science 330(6006), 916-917. 
Schade, S., Maué, P. and Davis, C., 2010. Collaboration in the Model Web. Workshop at Future Internet Symposium 2010: Towards Digital Earth: Search, Discover and Share Geospatial Data (FIS-DE 2010), Berlin, Germany, September 2010.

Schade, S. and Granell, C., 2012 . INSPIRE Fostering Innovation? Suggestion on a Living Lab Approach. INSPIRE 2012 Conference. Istanbul, Turkey, June 2012.

Shupeng, C. and van Genderen J., 2008. Digital Earth in support of global change research. International Journal of Digital Earth 1(1), 43-65.

Swart, R.J, Raskin, P., and Robinson, J., 2004. The problem of the future: sustainability science and scenario analysis. Global Environmental Change 14(2), 137-146.

United Nations Environment Programme (UNEP), 2012. GEO-5 Global Environment Outlook: Environment for the future we want. Valeta: UNEP.

Vaccari, L., Craglia, M., Fugazza, C., Nativi, S., Santoro, M., 2012. Integrative Research: The EuroGEOSS Experience. IEEE Journal of Selected Topics in Applied Earth Observations and Remote Sensing, in press.

Wiek, A., Farioli, F., Fukushi, K., and Yarime, M., 2012. Sustainability science: bridging the gap between science and society. Sustainability Science 7(S1), 1-4.

World Commission on Environment and Development (WCED), 1987. Our common future. Oxford: Oxford University Press.

Wright, D.J., and Wang, S., 2011. The emergence of spatial cyberinfrastructure. Proceedings of the National Academy of Sciences 108(14), 5488-5491.

Yang, C., Raskin, R., Goodchild, M.,and Gahegan, M., 2010. Geospatial cyberinfrastructure: past, present and future. Computers, Environment and Urban Systems 34 (4), 263-358.

Zheng, Z., Ke, G., Zeng, D., Ram, S. and Lu, H., 2011. Next-Generation Team-Science Platform for Scientific Collaboration. IEEE Intelligent Systems 26(6), 72-76. 
Figure 1. Introduction to the innovation space

Figure 2. Existing gap to leverage R\&D results to market-ready products (typology of open innovation strategies modified from Balloon et al. (2005))

Figure 3. Overall methodology to enable customer-driven product development (adapted from Apollon project)

Figure 4. Open innovation ecosystem for DELI 


\section{Users}

\section{Innovation Space}
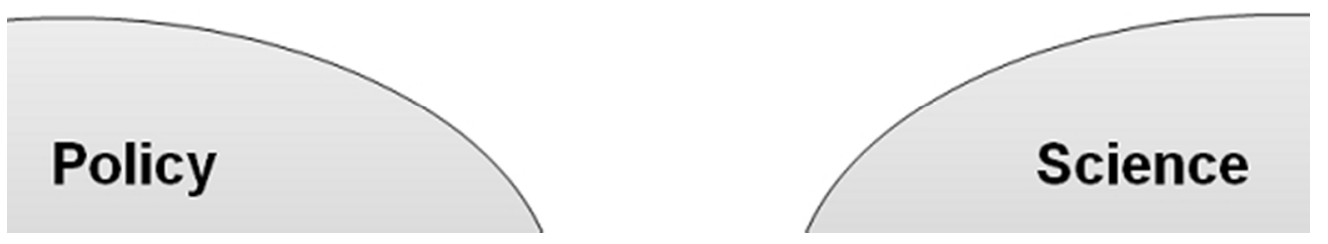

Introduction to the innovation space $158 \times 77 \mathrm{~mm}(96 \times 96 \mathrm{DPI})$ 

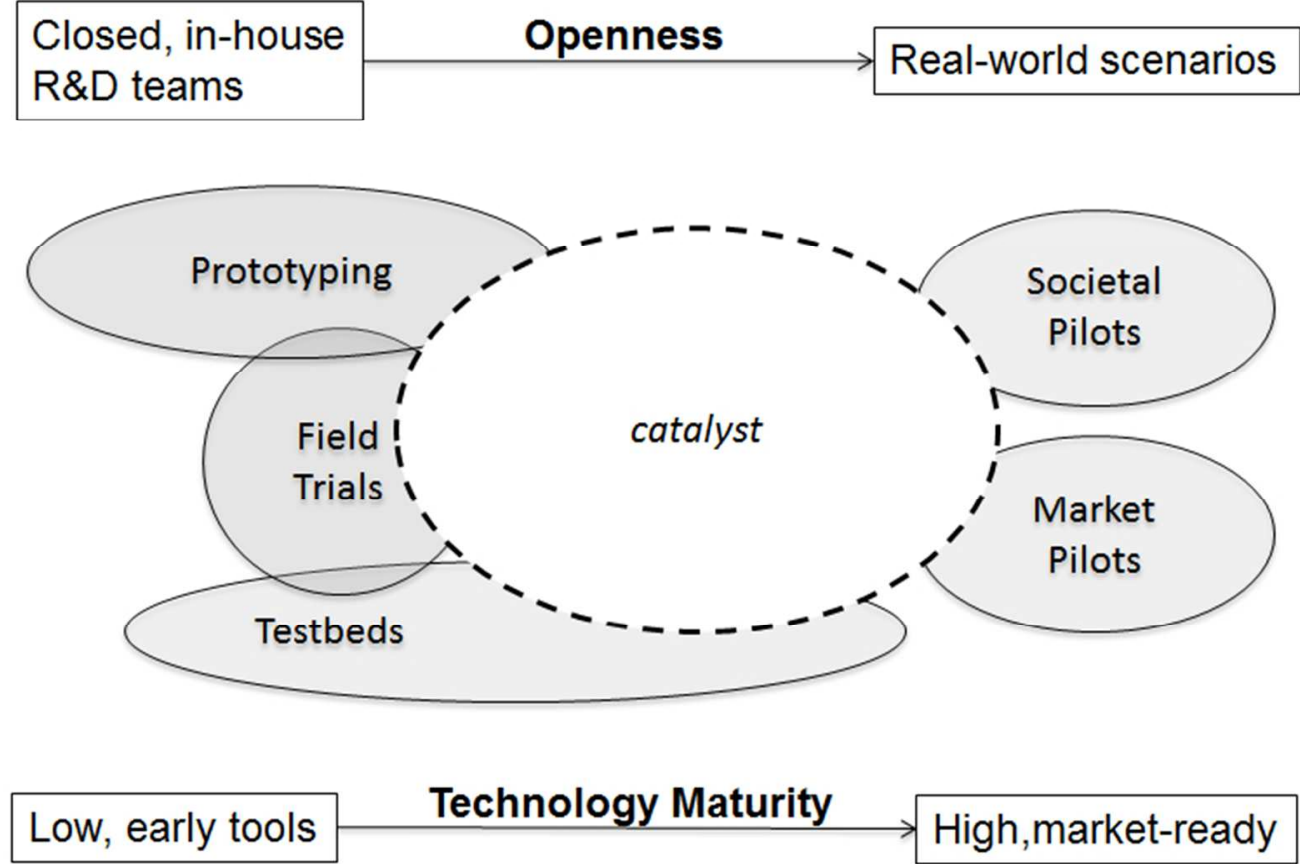

Existing gap to leverage R\&D results to market-ready products (typology of open innovation strategies modified from Balloon et al. (2005)) $198 \times 134 \mathrm{~mm}(96 \times 96 \mathrm{DPI})$ 
1

2

3

4

5

6

7

8

9

10

11

12

13

14

15

16

17

18

19

20

21

22

23

24

25

26

27

28

29

30

31

32

33

34

35

36

37

38

39

40

41

42

43

44

45

46

47

48

49

50

51

52

53

54

55

56

57

58

59

60

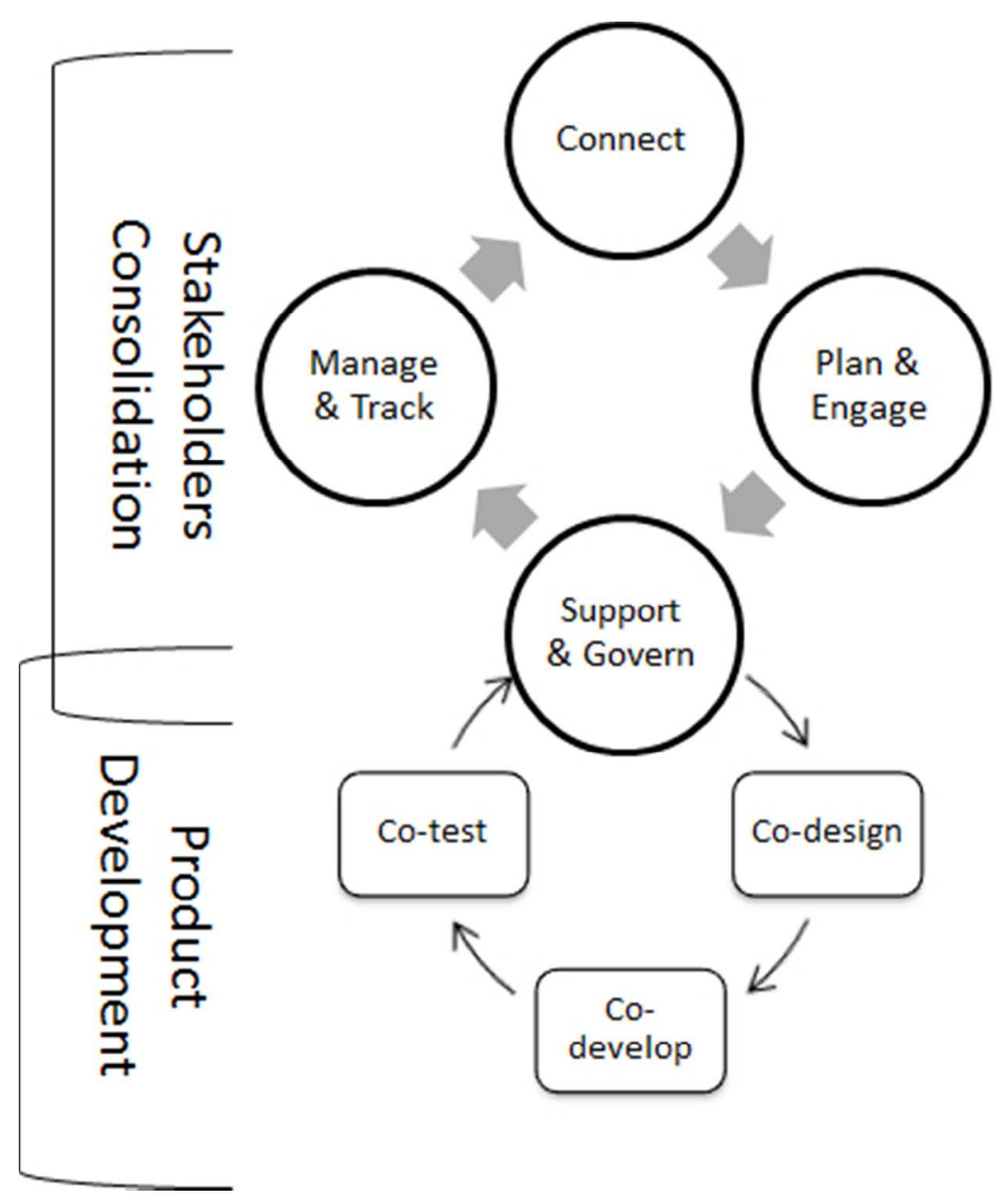

Overall methodology to enable customer-driven product development (adapted from Apollon project) $116 \times 126 \mathrm{~mm}(96 \times 96$ DPI) 


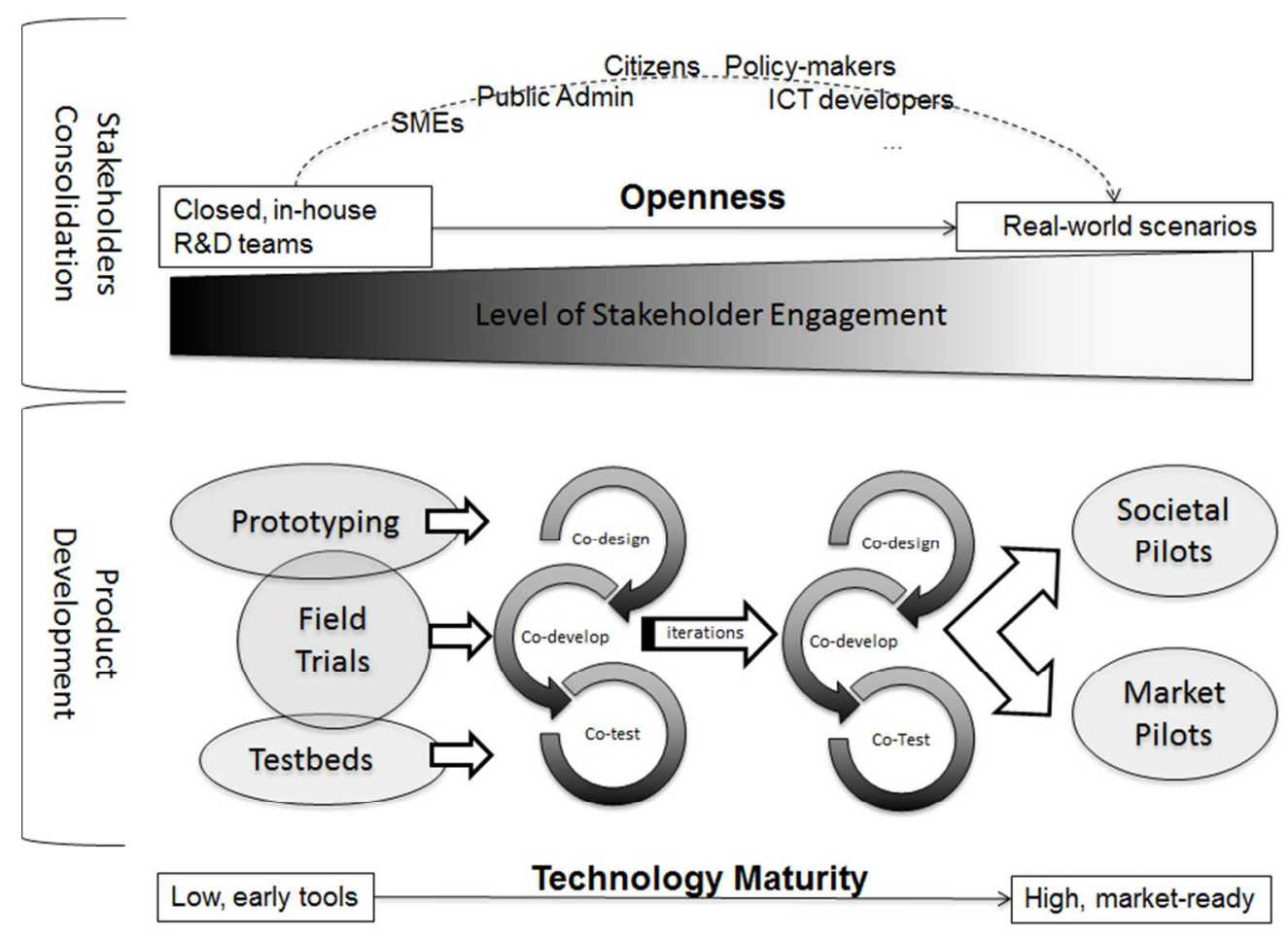

Open innovation ecosystem for DELI $230 \times 166 \mathrm{~mm}$ (96 x 96 DPI) 